\title{
MadGraph/MadEvent at Work: From QCD to New physics
}

\author{
Simon de Visscher * \\ Universite catholique de Louvain \\ Centre for Particle Physics and Phenomenology \\ 2, chemin du cyclotron, 1348 Louvain la Neuve, Belgium
}

\begin{abstract}
The Madgraph/MadEvent software is a powerful user-driven matrix element based event generator. Recently, different matching schemes have been implemented which allows to generate inclusive multi-jet samples by combining parton level events with parton showers consistently. This note presents results of the Modified MLM scheme applied on $t \bar{t}+$ jets samples generated for LHC with Madgraph/MadEvent. First, general tools of Madgraph/MadEvent are briefly described. Second, the matching method as well as his impact on key observables is presented. Feasability of evaluating theoritical uncertainties of simulation chains is also illustrated.
\end{abstract}

\section{MadGraph/MadEvent and tools: a complete chain of simulation.}

One of the challenges for exploring new physics sectors at Tevatron and LHC is to extract information from processes with highly complicated final states. In hadronic colliders, the understanding of multi-jets events is probably one of the most important tasks in order to be able to extract the rare non-SM information that could be possibly produced. Therefore the correct simulation of such processes is crucial.

MadGraph/MadEvent $[2,3](\mathrm{MG} / \mathrm{ME})$ is a user-driven matrix element based event generator for high-energy collisions simulation. The whole package is accessible by the web ${ }^{\mathrm{a}}$ or can be downloaded and run locally on user's machine or cluster. The role of MadGraph is to generate all amplitudes of relevant subprocesses given by the user. The list of Feynman diagrams is also computed. Beside a very high flexibility for handling new models, processes can have many particles in the final state. Currently, models implemented are SM, MSSM, 2HDM, HEFT and UED. There are also additional possibilities for testing a new model: starting from a Lagrangian with MadRules or by simply adding new particles and their interactions using UserModel. MadEvent uses as input the output of MadGraph, and produces a MonteCarlo package to calculate the cross section for a given process and also generate events. The architecture of the calculation code is intrinsically parallel and is therefore convenient to use with multi-CPU clusters. Several tools are available within the MG/ME package. To perform showering and hadronization, a standard version of Pythia [4] is available, and a generic detector simulation can be performed using the PGS software [5]. Both are installed on clusters accessible by the web. In addition, two analysis packages are available: MadAnalysis and ExRootAnalysis. The output of MadEvent is in "Les Houches" [6] standard format, which is compliant with external tools (CMSSW, Bridge [7],...). The $\mathrm{MG} / \mathrm{ME}$ package with tools therefore provides a complete chain of simulation, from the hard scale process, including signal and backgrounds in any model, down to detector simulation.

\footnotetext{
*I would like to thank Johan Alwall, Fabio Maltoni and Vincent Lemaitre for the great help they brought me all along this work.

${ }^{a}$ http://madgraph.phys.ucl.ac.be, http://madgraph.hep.uiuc.edu, http://madgraph.roma2.infn.it
} 
This is therefore a natural framework to perform complete analyses in several different theoretical contexts.

\section{Jet matching}

Matching techniques are needed to produce inclusive samples without double counting between subsamples. An additional problem is to be sure that no region of the phase space is forgotten.

As shown in the figure 1 , two families of matching co-exists. As main differences, reweighting of events is done using Sudakov terms and veto on parton showers in CKKW [8] matching schemes whereas MLM-based [9] schemes rejects events with jets unmatched with partons. In the CKKW case, partons are clustered in jets with the $K_{T}$ algorithm [10] while the original MLM method [11] uses a cone algorithm and minimum $P_{T}$ cut. The new method used here called Modified MLM matching scheme [12] can be viewed as a mixing of both families as it uses $K_{T}$ clustering and reject events with jets unmatched with partons (except in highest multiplicity subsamples). The

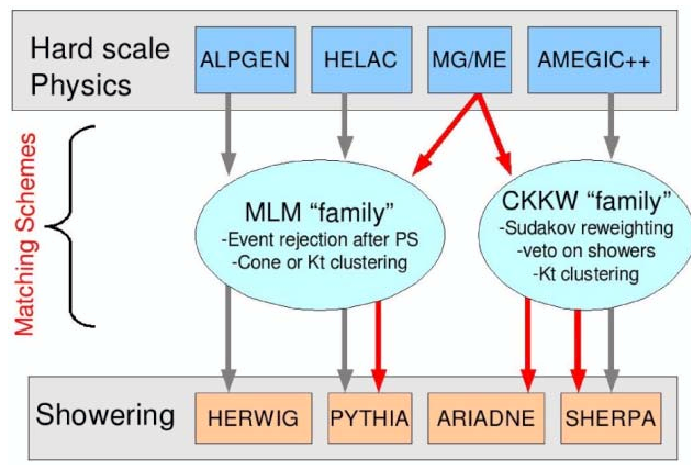

Figure 1: Flowchart of matching schemes between hard scale and showering simulators. results presented here concern that scheme tested with $t \bar{t}+$ jet(s) production for LHC. $\mathrm{W}+$ jets inclusive production has already been tested [12] and results will be published soon.

The modified MLM works as follows: first generate ME level events, with a minimal distance $d_{c u t}^{M E}$ in phase-space between partons ${ }^{\mathrm{b}}$. The beam is also taken into account. Second perform parton showers (PS) with the appropriate algorithm (here: Pythia 6.4). PS partons are then clustered in jets with $K_{T}$, using a maximal size of jet defined by a second cutoff $d_{c u t}^{P S}$. Finally the matching between ME partons and jets is realized. As the jet measurement is change when the showering is performed, $d_{c u t}^{M E}$ has to be smaller than $d_{c u t}^{P S}$. A factor 1.5 between both is reasonable. If the event is not of the highest multiplicity, each parton has to be matched with one jet, and vice versa. On the contrary, for highest multiplicity samples, an event with additional jets can be also kept.

\subsection{Differential jet rate}

The differential jet rate is a key variable to check if matching works properly. The transition between the two independent regions of the phase space (below and above the cutoff) has to be smooth and invariant with respect to the cutoff used. Differential jet rates $2 \rightarrow 1$ for

${ }^{\mathrm{b}}$ Distance in the phase space is defined here as

$$
d^{i j}=2 \min \left(P_{T}^{(i)}, P_{T}^{(j)}\right)^{2}\left[\cosh \left(\eta^{(i)}-\eta^{(j)}\right)+\cos \left(\phi^{(i)}-\phi^{(i)}\right)\right], \quad d^{\text {beam }}=P_{T}^{(i) 2}
$$


$t \bar{t}+0,1,2$ and 3 jets is shown in the figure 2 . The curves are related subsamples and their sum for different cutoffs. It appears that the transition is relatively smooth and matched shapes do not depend on the applied cut.
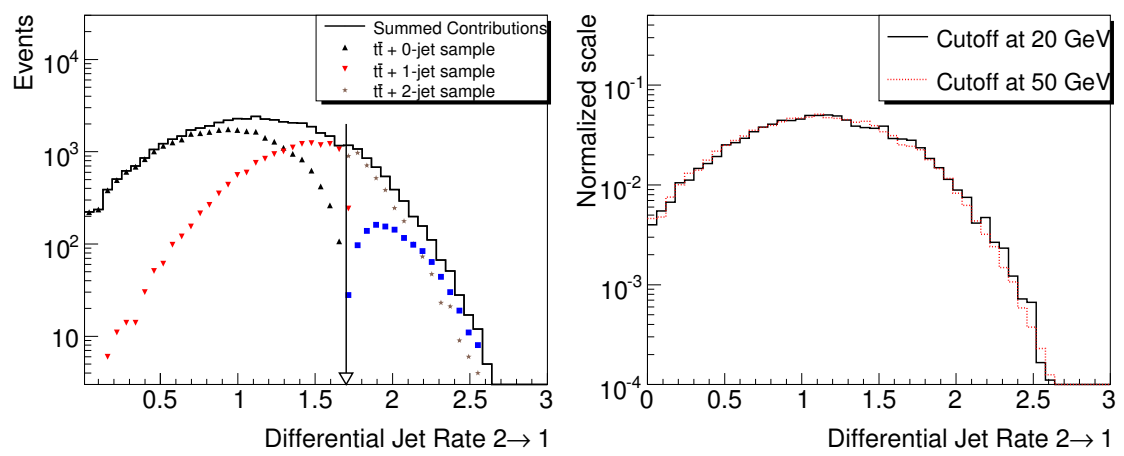

Figure 2: Differential jet rate $2 \rightarrow 1$ for $t \bar{t}+0,1,2$ and 3 jets for a cutoff of $50 \mathrm{GeV}$ indicated by an arrow (left). Summed contributions for two different cutoffs are also shown (right).

\subsection{Matching results}

The impact of extra-jets on top kinematics can be revealed with (among others) $\Delta \Phi$ angle between top quarks. This is illustrated on fig. 3 , where the curves are related to different subsamples as well as the sum of all contributions.

Those shapes can be compared with what as been obtained in ALPGEN (see [9]). The two matching schemes give very similar results even though the employed techniques are different.

The same holds for rapidity distributions of the leading jet as shown in the figure 4 . It appears that the use of Matrix-Element generators changes radically the kinematics of jets compared to what Pythia and Herwig standalone provide, which gives a strong motivation for using the matching procedure in multi-jet process generation.

\section{Conclusion}

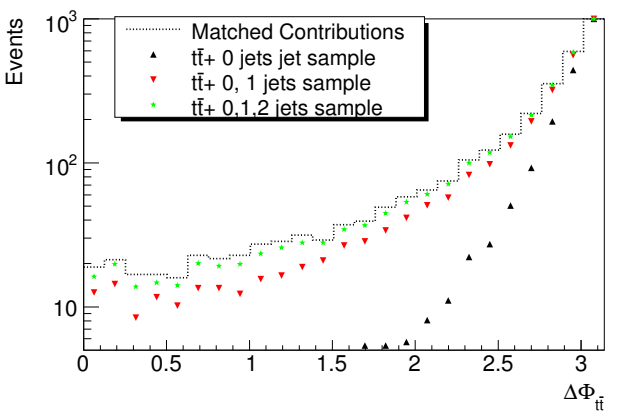

A test of Modified MLM matching implemented in the MadGraph/MadEvent generator and using Pythia for showering has been done on $t \bar{t}+$ jets samples. The evaluation of differential jet rates shows that this matching scheme is a very useful tool to prevent overlapping between phase spaces described by hard-scale generator and showering software used here. Comparison has been done with the original MLM matching scheme and results are similar, which provides a strong starting point for evaluation of systematic uncertainties related to 

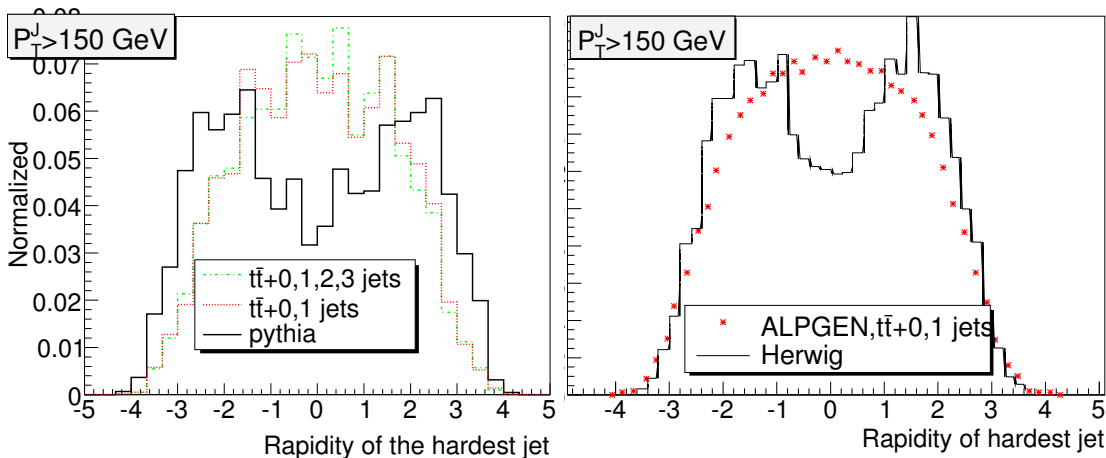

Figure 4: Rapidity of leading jet with $P_{T}^{\text {jet }}>150 \mathrm{GeV}$ obtained with MG/ME and Modified MLM matching (left) and ALPGEN and original MLM method (right). In addition distributions related to Pythia (left) and Herwig (right) standalone production are also illustrated

the simulation chain used. Moreover, this permits the generation of inclusive samples of top quark pair backgrounds at the LHC.

\section{References}

[1] Slides: http: //indico. cern. ch/contributionDisplay. py? contribId=210\&sessionId=6\&conf Id=9499

[2] F. Maltoni and T. Stelzer. Madevent: Automatic event generation with madgraph. JHEP, 02:027, 2003.

[3] J. Alwall et al. Madgraph/madevent v4: The new web generation. Submitted at JHEP in June 07.

[4] T. Sjostrand et al. Pythia 6.4 physics and manual. JHEP, 05:026, 2006.

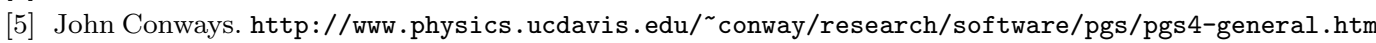

[6] J. Alwall et al. A standard format for les houches event files. Comput. Phys. Commun., 176:300-304, 2007.

[7] Patrick Meade and Matthew Reece. Bridge: Branching ratio inquiry/decay generated events, http://www.citebase.org/abstract?id=oai:arxiv.org:hep-ph/0703031, 2007.

[8] F. Krauss. Matrix elements and parton showers in hadronic interactions. JHEP, 0208:015, 2002.

[9] Michelangelo L. Mangano et al. Matching matrix elements and shower evolution for top-quark production in hadronic collisions. JHEP, 01:013, 2007.

[10] S. Catani et al. Longitudinally invariant $\mathrm{k}(\mathrm{t})$ clustering algorithms for hadron hadron collisions. Nucl. Phys., B406:187-224, 1993.

[11] Michelangelo Mangano. http://mlm.home.cern.ch/mlm/talks/lund-alpgen.pdf.

[12] J. Alwall et al. Comparative study of various algorithms for the matching of parton showers and matrix elements in hadronic collisions. to be published. 